\title{
On the quantum gravity and the stationary Universe
}

\author{
Koryukin V.M. ${ }^{1}$, Koryukin A.V. ${ }^{2}$ \\ ${ }^{1}$ Mari State University, Yoshkar-Ola, Russia; \\ ${ }^{2}$ Kazan Federal University, Kazan, Russia; \\ E-mail: Koryukin <vmkoryukin@gmail.com>;
}

We propose the quantum theory for the description of gravitation interactions both at large distances and at small ones. As a result it is appeared the scope for the physical interpretation of "the absolute cosmological principle" and the construction of the stationary Universe.

Keywords: absolute cosmological principle, dark matter, neutrinos, quantum gravity.

DOI: $10.18698 / 2309-7604-2015-1-220-229$

It is well known that the classical physical theory must be the consequence of the quantum theory. It is precisely therefore so much effort was made for the construction of the quantum gravity. We propose the variant of its construction, which did not find an alternative.

The principle of the theoretical notions adequacy to experimental data must be put in the base of the serious physical theory. It is precisely therefore we attach the fundamental importance to symmetries which's reflecting the matter properties in the condensed (pithy) form. For this in the elementary particle physics is used the scattering matrix which allows to guess a form of transition operators if only for linear approximation. Because we must forecast results of future experiments, the description of physical systems states will proceeds by use of smooth functions, which it is desirable to obtain as solutions of differential equations. It is precisely therefore we shall approximate the transition operators by differential operators using the variation formalism. We note that the presence of the Universe neutrino background with the finite Fermi energy $E_{F}$ is the catalytic agent of stochastic processes, but the large value of this energy causes to the determinancy of physical processes (we shall use the system of units $h /(2 \pi)=c=1$, where $h$ is the Planck constant and $c$ is the velocity of light). Specifically we connect the large value of the Fermi energy and the low temperature of the Universe neutrino background with the stability (or if only with the metastability) of elementary particles.

In the degenerate state background fermions of Universe, generating Fermi and Bose liquids, are weakly-interacting particles, but it is not excluded by the interaction with hadrons their exhibition as color fermions - ghosts. We do not exclude also the possibility, that in the state of the Fermi liquid with the sufficiently low ( $T_{\mathrm{o}} \ll<m_{p}, m_{p}$ is the proton mass) temperature $T_{\mathrm{o}}$, (that must be characterized by the spontaneously broken symmetry) they must be considered as right 
neutrinos and left antineutrinos with the sufficiently high $\left(m_{p}<<E_{F}\right)$ Fermi energy $E_{F}$ ("sterile" neutrinos and "sterile" antineutrinos [1]). It must be exhibited in the absence of these particles by decays attributed to weak interactions of low energies (a mirror asymmetry) [1]. Note, that the transition to the classical physics proceeds by use of $E_{F} \rightarrow \infty$. We shall consider the covariant gauge theory of strong interactions in the affine connection space, on the base of which may be produced both the quantum chromodynamics and the strong gravitation theory [2]. We regard, that the standard gravitation interaction is not the fundamental one, but it is generated by collective oscillations in the Universe neutrino sea [3]. In consequence of this the weak interaction acquires the global value, because it causes the bond of neutrinos both one with the other and with charged leptons and quarks.

We consider the action which has the form as the following integral

$$
A=\int_{\Omega_{n}} \Lambda d_{n} V=\int_{\Omega_{n}} \kappa \bar{X}(\Psi) \rho X(\Psi) d_{n} V
$$

( $\Lambda$ is a Lagrangian). Here and further $\kappa$ is a constant; $\rho=\rho(x)$ is the density matrix (tr $\rho=1, \rho^{+}$ $=\rho$, the top index " + " is the symbol of the Hermitian conjugation) and the bar means the generalized Dirac conjugation which must coincide with the standard one in particular case that is to be the superposition of Hermitian conjugation and the spatial inversion of the space-time $M_{4}$. We shall name solutions $\Psi(x)$ of differential equations, which are being produced by the requirement of the minimality of the integral (1), as the maximum plausible realizations of Lie local loops $G_{r}(x)$ [2] and we shall use for the construction of the all set of functions $\{\Psi(x)\}$ (generated by the help of the transition operators).

Let $E_{n+N}$ is the vector fiber space with the base $M_{n}$ and the projection $\pi_{N}, \Psi(x)$ is the arbitrary section of fibre bundle $E_{n+N}, \nabla_{i}$ is the covariant derivative symbol. Let us to consider the infinitesimal substitutions defining the vector space mapping of the neighbour points $x$ and $x+\delta x\left(x \in U, \quad x+\delta x \in U, U \subset M_{n}\right)$ and conserving the possible linear dependence between vectors. We demand that the action A was the invariant one with respect to the infinitesimal substitutions of the local Lie loop $G_{r}(x)$ conserving the type of geometrical objects. By this the components $C_{a b}^{c}(x)$, alternating on down indices of the structural tensor, must satisfy to the generalized Jacobi identities 


$$
C_{[a b}^{d} C_{c] d}^{e}-\xi_{[a}^{i} \nabla_{|i|} C_{b c]}^{e}+\xi_{[a}^{i} \xi_{b}^{j} R_{|i j| c}^{e}=0
$$

$\left(R_{i j c}{ }^{e}(x)\right.$ are the curvature tensor components of the connection $\Gamma_{i a}^{b}(x)$; here and further $x^{i}$ are the co-ordinates of the point $x ; x^{i}+\delta x^{i}$ are the co-ordinates of the point $x+\delta x$; Latin indices $a$, $b, c, d, e$ will run the values of integers from 1 to $\mathrm{r}$; Latin indices $i, j, k, \ldots$ will run the values of integers from 1 to $n$ ).

That the appearance of stringent restrictions can be excluded on a Lagrangian we introduce its dependence on gauge fields $B(x)[2]$. Let

$$
B B^{+}=\rho \operatorname{tr}\left(B B^{+}\right)
$$

in the Lagrangian (1). Further fields $\Psi(x)$ we shall name as prime ones. We denote the components of the gauge fields $B(x)$ as: $B_{a}^{c}(x)$. Probably the rank of the density matrix $\rho$ equals $n$, but it is impossible to eliminate that the given equality is satisfied only approximately when some components of a density matrix can be neglected. In any case we shall consider that among fields $B_{a}^{b}$ the mixtures $\Pi_{a}^{i}$ were formed with non-zero vacuum means $h_{a}^{i}$ which determine differentiable vector fields $\xi_{a}^{i}(x)$ for considered domain $\Omega_{n}$ as:

$$
\Pi_{a}^{i}=B_{a}^{b} \xi_{b}^{i}
$$

(spontaneous breaking of symmetry, fields $\xi_{a}^{i}(x)$ determine a differential of a projection $d \pi$ from $\Omega_{r} \subset M_{r}$ in $\left.\Omega_{n} \subset M_{n}\right)$.

Let us rewrite the integral (1) in the following manner

$$
\mathrm{A}_{t}=\int_{\Omega_{n}} \Lambda_{t} d_{n} V=\int_{\Omega_{n}}\left[\Lambda_{\circ}+\Lambda_{1}(\Psi)+\Lambda_{2}(B)\right] d_{n} V
$$

where $\Lambda_{\circ}$ is the constant which is connected with the normalization and 


$$
\begin{gathered}
\Lambda_{1}=\kappa \overline{X^{b}}(\Psi) \rho_{b}^{a} X_{a}(\Psi)=\kappa \overline{D^{a}} \Psi D_{a} \Psi /\left(B_{b}^{+c} B_{c}^{b}\right), \\
D_{a} \Psi=-B_{a}^{c} X_{c}(\Psi)=B_{a}^{c}\left(\xi_{c}^{i} \nabla_{i} \Psi-L_{c} \Psi\right) .
\end{gathered}
$$

Since the action (5) must be invariant by infinitesimal substitutions of the Lie local loop $G_{r}(x)$, then the Lagrangian $\Lambda_{2}(B)$ must depend on the gauge [1] (boson) fields $B(x)$ by intensities $F_{a b}^{c}(B)$, having the form

$$
F_{a b}^{c}=\Theta_{d}^{c}\left(\Pi_{a}^{i} \nabla_{i} B_{b}^{d}-\Pi_{b}^{i} \nabla_{i} B_{a}^{d}+\Xi_{a b}^{d}\right)
$$

Where

$$
\Theta_{b}^{c}=\delta_{b}^{c}-\xi_{b}^{i} \Pi_{i}^{a}\left(B_{a}^{c}-\beta_{a}^{c}\right), \quad \Xi_{a b}^{e}=B_{d}^{e}\left(B_{a}^{c} L_{c b}^{d}-B_{b}^{c} L_{c a}^{d}\right)-B_{a}^{c} B_{b}^{d} C_{c d}^{e}
$$

Hereinafter a selection of fields $\Pi_{i}^{a}$ and $\beta_{c}^{a}$ are limited by the relations:

$$
\Pi_{j}^{a} \Pi_{a}^{i}=\delta_{j}^{i}, \quad \beta_{c}^{a} \xi_{a}^{i}=h_{c}^{i}
$$

Further it is convenient to use the following Lagrangian:

$$
\Lambda_{2}=\frac{\kappa_{\circ}^{\prime}}{4} F_{a b}^{c} F_{g e}^{d}\left[t^{a g}\left(s_{c}^{e} s_{d}^{b}-v s_{c}^{b} s_{d}^{e}\right)+t^{b e}\left(s_{d}^{a} s_{c}^{g}-v s_{c}^{a} s_{d}^{g}\right)+u_{c d}\left(t^{a g} t^{b e}-v t^{a b} t^{g e}\right)\right]
$$

$\left(\kappa_{\circ}^{\prime}, v\right.$ are constants) [1]. If $s_{a}^{b}=\delta_{a}^{b}, t^{a b}=\eta^{a b}, u_{a b}=\eta_{a b}\left(\eta_{a b}\right.$ are metric tensor components of the flat space and $\eta^{a b}$ are tensor components of a converse to basic one) then the given Lagrangian is most suitable one at the description of the symmetry matter (all matter states are equally likely), because it is most symmetrical one concerning intensities of the gauge fields $F_{a b}^{c}$ (within hadrons and within so named "black holes"). What is more we shall require the realization of the 
correlations:

$$
L_{c d}^{a} \eta^{d b}+L_{c d}^{b} \eta^{d a}=0
$$

that the transition operators $L_{a c}^{b}$ generate the symmetry which follows from the made assumptions. The transition to the matter description of the observable space region for which one it is possible to suspect that the presence of cluster states of interacting particles will be expressed in following formula for tensors $s_{a}^{b}, t^{a b}, u_{a b}$ and $h_{i}^{a}$ :

$$
\begin{gathered}
s_{a}^{b}=s \xi_{a}^{i} h_{i}^{b}+\xi_{a}^{\underline{c}} \varepsilon_{\underline{c}}^{b}, \quad t^{a b}=t \varepsilon_{(I)}^{a} \varepsilon_{(k)}^{b} \eta^{(I)(k)}+\varepsilon_{\underline{\varepsilon^{\prime}}}^{a} \varepsilon_{\underline{d}}^{b} \eta^{\underline{c} \underline{d}}, u_{a b}=u \xi_{a}^{i} \xi_{b}^{j} h_{i}^{c} h_{j}^{d} \eta_{c d}+\xi_{a}^{\underline{c}} \xi_{b}^{\underline{d}} \eta_{\underline{\underline{d}} \underline{ }}, \\
h_{i}^{a}=h_{i}^{(k)} \varepsilon_{(k)}^{a} .
\end{gathered}
$$

$((i),(j),(k),(l), \ldots=1,2, \ldots, n ; \underline{a}, \underline{b}, \underline{c}, \underline{d}, \underline{e}=n+1, n+2, \ldots, n+\underline{r} ; \underline{r} / r \ll 1)$, where fields $h_{i}^{(j)}(x)$, taking into account the relations (13), are determined uniquely from equations: $h_{k}^{a} h_{a}^{i}=\delta_{k}^{i}$. Similarly tensors $\eta^{(i)(j)}, \eta^{\underline{a b}}$ are determined from equations: $\eta^{(i)(k)} \eta_{(j)(k)}=\delta_{(j)}^{(i)}, \eta^{\underline{a b}} \eta_{\underline{c b}}=\delta_{\underline{c}}^{\underline{a}}$, while tensors $\eta_{(i)(j)}, \eta_{\underline{a b}}$ are determined as follows: $\eta_{(i)(k)}=\eta_{a b} \varepsilon_{(i)}^{a} \varepsilon_{(k)}^{b}, \eta_{\underline{a b}}=\eta_{c d} \varepsilon_{\underline{a}}^{c} \varepsilon_{\underline{b}}^{d}$. We shall connect constants $\varepsilon_{(i)}^{a}, \varepsilon_{\underline{b}}^{a}$ with a selection of the gauge fields $\Pi_{i}^{a}(x)$ recording them by in the form

$$
\Pi_{i}^{a}=\varepsilon_{(k)}^{a} \Phi_{i}^{(k)}+\varepsilon_{\underline{b}}^{a} \mathrm{P}_{i}^{\underline{b}}
$$

and let $\varepsilon_{\underline{b}}^{a}=0$. Besides we shall apply the decomposition of fields $B_{b}^{a}(x)$ in the form

$$
B_{c}^{a}=\zeta_{i}^{a} \Pi_{c}^{i}+\zeta_{\underline{b}}^{a} A_{c}^{\underline{b}}
$$

where $A_{c}^{\underline{b}}=\xi_{a}^{\underline{b}} B_{c}^{a}$. Note that we decompose the physical system described by fields $B_{b}^{a}(x)$ on two subsystems. One of them described by fields $\Pi_{a}^{i}(x)$, will play the role of the slow subsystem. In addition components of intermediate tensor fields $\xi_{a}^{i}(x), \xi_{a}^{\underline{b}}(x), \zeta_{i}^{a}(x), \zeta_{\underline{b}}^{a}(x)$ should be 
connected by the relations: $\zeta_{i}^{a} \xi_{a}^{j}=\delta_{i}^{j}, \zeta_{i}^{a} \xi_{a}^{\underline{b}}=0, \zeta_{\underline{b}}^{a} \xi_{a}^{j}=0, \zeta_{\underline{b}}^{a} \xi_{a}^{\underline{c}}=\delta_{\underline{b}}^{\underline{c}}$. So, we shall use the reduced set of fields $\left\{\Pi_{c}^{i}(x), A_{c}^{\underline{b}}(x)\right\}$ instead of the full set $\left\{B_{c}^{a}(x)\right\}$.

Of course, taking into account the absence of a bijection between the real world and the mathematical one, we can construction the maximum plausible physical theory only. It allows using an elemental description, if only for a local domain. We shall use that smooth manifolds are locally diffeomorphic ones to the Euclidean space or to the pseudo-Euclidean space in a certain neighborhood of any point. Therefore we shall choose the connection components $\Gamma_{i a}^{b}(x)$ equal to zero in the region under consideration. Since stable states or metastable states are characterized the specific symmetries, then giving the parameter dependence of structural tensor components $C_{a b}^{c}$, we can describe decay processes of elementary particles if only approximately. Specifically, we shall consider that the process of the spontaneous symmetry breaking is characterized the quasigroup structure (we take account of the presence of the Universe neutrino background which is the catalytic agent of stochastic processes, including decays of elementary particles). In consequence of this it is logically connect the stability of differential equations (2) solutions with the stability of elementary particles. As a result functions $C_{a b}^{c}(x)$ must describe the process of spontaneous breaking of symmetry at hadrons decay. Specifically, when $n=r=8$, it allows to do not increase the count of gauge fields beyond 8 as in the grand unified theory. Thereby we consider that gluons are present in the space domain where intermediate vector bosons are absent and on the contrary intermediate vector bosons are present in the space domain where gluons are absent.

Further we shell rely on the cold plasma theory developed for the first-kind superconductor and for the second-kind superconductor. By this a nuclear matter is an analog of second-kind superconductors with respect to gluons, which's as vortices penetrate in a Bose condensate of Cooper pairs compounded from neutrinos, at the same time the vacuum with respect to gluons is an analog of first-kind superconductors. We assume that the interaction energy must depend on a number of particles and quasi-particles participating in this interaction, defining its dependence to space coordinates by means of a mean number of bosons, which's are exchanged two hadrons. As a result ( $n$ is a number of bosons):

$$
E=-2 \int\left\{\sum_{\left(\sigma_{1}, \sigma_{2}\right)}\left[\left(\rho_{1} \sigma_{1} \rho \sigma_{1} \rho_{2} \sum_{n=0}^{N} n e^{-2 n \rho \sigma_{1} r / T}\right) / T \sum_{n=0}^{N} e^{-2 n \rho \sigma_{1} r / T}\right]\right\} d V_{1} d V_{2}
$$


where $\rho_{1}$ is the energy density of first body particles; $\rho_{2}$ is the energy density of second body particles; $\rho$ is the energy density of quasi-particles, $T$ is theirs temperature; $\sigma_{1}, \sigma_{2}$ are the crosssections with the emission or the absorption of particles or quasi-particles.

Because the gravitation theory is constructed, then naturally we must state the collision integral (16), retaining only two summands, as

$$
\begin{aligned}
& E=-2 \int\left[\left(\rho_{1} \sigma_{s 1} \rho \sigma_{s 2} \rho_{2} \sum_{n=0}^{N} n e^{-2 n \rho \sigma_{s 1} r / T}\right) / T \sum_{n=0}^{N} e^{-2 n \rho \sigma_{s 1} r / T}\right] d V_{1} d V_{2}, \\
& E=-2 \int\left[\left(\rho_{1} \sigma_{w 1} \rho \sigma_{w 2} \rho_{2} \sum_{n=0}^{N} n e^{-2 n \rho \sigma_{w 1} r / T}\right) / T \sum_{n=0}^{N} e^{-2 n \rho \sigma_{w 1} r / T}\right] d V_{1} d V_{2},
\end{aligned}
$$

where $\sigma_{s 1}, \sigma_{s 2}$ are the cross-sections with the gluon emission or with the gluon absorption (the strong gravitation), $\sigma_{w 1}, \sigma_{w 2}$ are the cross-sections with the quasi-particle emission or with the quasi-particle absorption in the neutrino collinear beam (spin waves), cementing together two hadrons and produced of the weak interaction (the standard weak gravitation) [3]. Hence (considering, that $N \rightarrow \infty$ ) it can obtain the potential as

$$
U(r)=-\frac{C_{s}}{e^{B_{s} r}-1}-\frac{C_{W}}{e^{B_{w} r}-1}
$$

$\left(B_{s} \propto \sigma_{s 1}, C_{s} \propto \sigma_{s 1} \sigma_{s 2}, \quad B_{w} \propto \sigma_{w 1}, C_{w} \propto \sigma_{w 1} \sigma_{w 2}\right.$ ) for the description of the gravitation interaction. Naturally, that at very small distances processes must be described the quantum chromodynamics, but not the strong gravitation theory.

Note that in the more general case, when the connection components $\Gamma_{i_{a}}^{b}(x)$ are not equal to zero and the Lie local loop $G_{r}(x)$ operates in the space of the affine connection as transitively so and effectively, then the correlations (2) become in the Ricci identity $\left(C_{a b}^{c}=2 S_{a b}^{c}, S_{a b}^{c}\right.$ are the torsion tensor components of the space $M_{r}$ ). Because the symmetry, characterizing the physical system, is selected in terms of experimental data, the geometrical structure is only the maximum plausible one. Hence it follows that it is desirable to use the spaces of the affine connection with the torsion for the description of particles. What is more precisely the torsion must depend on a rest mass of a particle. 
The assumption on the "sea" of quarks in the ground state allows using the Landau theory of the Fermi liquid considering observable particles as quasi-particles on the background of "sterile" neutrinos and "sterile" antineutrinos. The properties of the latter's must define the geometrical and topological properties of the space-time $M_{n}$. The transition to the description of the slow subsystem with the help of the space-time manifold is carried when the Fermi energy $\varepsilon_{F}$ of "sterile" neutrinos tends to infinity that allows using the Minkowski space in the capacity of the space characterizing the vacuum. In this case the quotation-marks in the words "sterile" neutrinos can be discarded, because these neutrinos will not collide with the other particles even at very high energies of the latter. The Universe all matter must be characterized (as it is adopted in the statistical physics) by the statistical sum with which it is a necessary to connect the cosmological term of General Relativity and it must not be equaled to zero. As a result, taking empirical data by the observation of type Ia supernovas into account, it understands the hypothesis necessity for the availability in the Universe of "dark energy", connecting it with the cosmological term.

We suggested considering "black holes" as hadrons with very large baryonic charges [4]. It allows simulating similar objects in laboratory conditions (at high energy accelerators). Naturally, that we connect the use necessity of the quantum chromodynamics in the cosmology with the chance of the processes explanation which's go in quasars and nuclei of Seyfert galaxies with the very large energy release. The Einstein theory cannot apply for this as in it the substantial object - the space-time torsion is absent by the gravitation geometrization (the space-time torsion is the locally diffeomorphic one to the corresponding structural tensor field of the Lie local loop characterizing the symmetry of the quantum system). What is more, in the Hawking process of the "black holes" quantum evaporation is violated the conservation low of the baryonic charge, accumulated the massive collapsing star. If we shall apply this process for the description to twophoton decay of pseudoscalar neutral mesons (this low takes place), then we receive a discrepancy with experimental data (instead the increase of the particles lifetime it is reducing with the growth of their masses).

We shall adhere to the principle which requires that the theoretical notions and statements were in agreement with experimental data. It is precisely therefore fundamental properties of a matter must find the representation in space properties. In the first place it is the quantization of electric charges. Secondly it is the space asymmetry of weak interactions. Moreover it is the stability or the metastability of physical systems. In consequence of this we put to doubt the standard cosmological model in which the Universe is supposed all but empty one. The Landau theory of Fermi liquid is the base for our approach. In this theory fermions forming of Fermi liquid 
are not considered and only quasi-particles bear the key responsibility for quantitative calculations. At present it is considered that Hubble discovered accidentally the Universe expansion law because he received the reddening in the under study spectrum of the emission for the majority of galaxies at distances from Earth to $10 \mathrm{Mpc}\left(1 \mathrm{Mpc} \approx 3.1 \cdot 10^{24} \mathrm{~cm}\right)$. Later the given region of Universe turned to be named as "Local volume" and the recession of galaxies turned to be named as the Hubble flux which is bounded with the cosmological expansion. Note that if distances from Earth are not the more $50 \mathrm{Mpc}$ then the region (which coincides with "the homogeneity cell" of the Universe) is named as "the Local Universe".

We divide the Universe matter into two subsystems (slow and rapid), so that the baryon matter (a rapid subsystem) behavior (a rapid subsystem) must be defined by the non-baryon matter (a slow subsystem) behavior. Just rapid subsystem particles allow doing predictions and conclusions to us on states and properties of particles of the Universe matter slow subsystem (a dark matter), using methods of inverse problems. The presence of a dark matter was detected for the first time in the research of the galaxies motion which must be caused the gravitation interaction and it is just that effect which allows detecting the presence of a Bose condensate. Since in our approach it is laid stress on known particles, then we have the weighable evidence of the presence of Cooper pairs of Universe background neutrinos. We can use the similar mechanism to Bardeen-Cooper-Schrieffer theory of superconductivity for the formation explanation of Cooper neutrino pairs the more so that a dark matter is detected only in the vicinity of the University baryon matter. At the same time on scales over $1 M p c$ must be observed the reverse process of Cooper neutrino pairs decay. This must causes to the increase of the background neutrino density in the Fermi liquid and to the expansion of the given volume of the Universe (Hubble flux of "Local volume").

All this allows return to "the absolute cosmological principle" (the Universe must look equally as in any points and in any directions and also at any instants of time [5]) the more so, that at very large distances in consequence of (18) the gravitation interaction can be ignored. Thus we can continue the construction of the stationary Universe on scales exceeding "the homogeneity cell", for which it can keep the theory developed in the standard model. As a result it is become a highpriority test of the hypothesis on the presence of the Universe neutrino background under laboratory conditions.

V.M. Lobashev [6] marked the beginning of this, investigating parameters, characterizing the decay of the tritium. Instead of the expected crevasse in the energy spectrum of electrons escaping from tritium nuclei (that gives the opportunity to prove the presence of the neutrino rest 
mass) was observed the stable plateau. The received data allowed to him to do the conclusion on the presence of the pronounced neutrino cloud ambient our galaxy and also on the solar neutrino rays. Therefore it might be worthwhile to make experiments for the observation of the neutrino background temperature; it is possible with the employment of the Josephson effect. Of course, technical problems are considerable and first of all they are connected with the screening from the electromagnetic radiation but the result will have the fundamental importance. Particularly if by monitoring the temperature waves will be detected.

\section{References}

1. Koryukin V.M. (2008). The confinement and the dark matter of the Universe. Proceedings of the XIX International Baldin Seminar on High Energy Physics Problems: Relativistic Nuclear Physics and Quantum Chromodynamics, JINR, Dubna, V. 1, XVI, 61-66.

2. Koryukin V.M. (1990). The strong gravitation and the chromodynamics. Sov. J. Nucl. Phys., Vol. 52, 573 - 579.

3. Koryukin V. (1992), Gravitation and Weak Interactions in the Gauge Fields Covariant Theory. Proceedings of III International Symposium on Weak and Electromagnetic Interactions in Nuclei, Singapore, New Jersey, London, Hongkong: World Scientific, 456 - 458.

4. Koryukin V.M. (2013). Two subsystems of the Universe matter and the "black holes". Proceedings of International Scientific Meeting "Physical Interpretations of Relativity Theory - 2013”, Moscow: BMSTU, 163-169.

5. Weinberg S. (1972). Gravitation and Cosmology. New York: John Wiley.

6. Lobashev V.M. (2000). Direct Search for the Neutrino Mass in the Beta-Decay of Tritium. Status of the "Troitsk v-Mass” Experiment. Physics of Atomic Nuclei, Vol. 63, № 6, 1037-1043. 